\title{
Excess manganese as the origin of the low-temperature anomaly in NiMnSb
}

\author{
B. Zhang, ${ }^{1}$ J. A. Heuver, ${ }^{2}$ F. Wang, ${ }^{3}$ J. Baas, ${ }^{2}$ G. A. de Wijs, ${ }^{1}$ T. Fukuhara, ${ }^{3}$ T. T. M. Palstra, ${ }^{2}$ and R. A. de Groot ${ }^{1,2, *}$ \\ ${ }^{1}$ Radboud University Nijmegen, Institute for Molecules and Materials, Heyendaalseweg 135, 6525 AJ Nijmegen, The Netherlands \\ ${ }^{2}$ Solid State Materials for Electronics, Zernike Institute for Advanced Materials, University of Groningen, \\ Nijenborgh 4, 9747 AG Groningen, The Netherlands \\ ${ }^{3}$ Toyama Prefectural University, Department of Liberal Arts and Science, Imizu, Toyama 939-0398, Japan
}

(Received 18 February 2013; published 18 July 2013)

\begin{abstract}
The archetype of half-metallic magnetism, $\mathrm{NiMnSb}$, has been reported to show an anomaly at low temperature. The high degree of spin polarization of the conduction electrons, characteristic of a half metal, is lost above this temperature. Recently reported experiments show that this anomaly is not an intrinsic property of NiMnSb: it requires an excess of (interstitial) manganese. Electronic structure calculations reported here show that the excess manganese orders antiferromagnetically with respect to the host magnetization, reduces the half-metallic band gap, and pushes the top of the valence band up to $36 \mathrm{meV}$ below the Fermi level. Thermal excitations from minority to majority spin channel induce an avalanche effect, leading to the disordering of the magnetic moments of the excess manganese. This mechanism is supported by measurements of the magnetization as a function of temperature on $\mathrm{NiMn}_{1.05} \mathrm{Sb}$ : It shows a maximum in the magnetization measured in a field of 400 Oe.
\end{abstract}

DOI: 10.1103/PhysRevB.88.014418

PACS number(s): 75.50.Cc, 71.55.Ak, 85.75.-d

\section{INTRODUCTION}

$\mathrm{NiMnSb}$ is the first example of a half metal, ${ }^{1}$ and its potential in what is known as a spintronic device ${ }^{2}$ was recognized from the beginning. ${ }^{3}$ The half-metallic properties in the bulk were confirmed experimentally by spin-resolved positron annihilation, ${ }^{4,5}$ but surfaces and interfaces of half metals turned out to be delicate. ${ }^{6-8}$ A complication specific for NiMnSb is the low temperature anomaly: ${ }^{9}$ a crossover in the temperature dependence of both magnetization and resistivity (the magnetization, $M(T) \propto T^{1.5}$ below $80 \mathrm{~K}$, while $M(T) \propto T^{2.0}$ above $100 \mathrm{~K}$; the resistivity, $\rho(T) \propto T^{\alpha}$ below $50 \mathrm{~K}$ with $1.7<\alpha<2.2$, while $\rho(T) \propto T^{\beta}$ above $100 \mathrm{~K}$ with $1.3<\beta<1.5$ ). The first observation ${ }^{10}$ reports a strong increase in Mn and Ni magnetic moments upon cooling below $90 \mathrm{~K}$ in thin films. Thermal excitations ${ }^{11}$ could be responsible for the loss of high spin polarization, possibly in the form of a noncollinear ordering of the magnetic moment on $\mathrm{Ni}$ with respect to the $\mathrm{Mn},{ }^{12}$ but others considered thermal excitation unlikely in stoichiometric NiMnSb (the position of the Fermi level with respect to the top edge of the valence bands of the minority spin is too high). ${ }^{13}$ Many possible defects were considered, like antisite defects, ${ }^{14,15}$ but in many of the cases with reasonable formation energies the half-metallic properties were conserved. An alternative explanation is the occurrence of nonquasiparticle states, ${ }^{16}$ but the underlying many-body theory requires a Hubbard $\mathrm{U}$ (about $2 \mathrm{eV}$ ) of more than an order of magnitude in excess of the experimentally derived value (about $0 \pm 0.1 \mathrm{eV}$ in the isostructural and isoelectronic $\mathrm{PtMnSb}^{13}$ ). The most common method to synthesize bulk Heusler alloys is arc-melting stoichiometric amounts of high purity elements. ${ }^{17}$ The synthesis of stoichiometric NiMnSb is hindered by the large variation in vapor pressure of the constituent elements at high temperature. Recently the situation changed, when experiments using samples synthesized under controlled partial pressures, showed that the low temperature anomaly is not an intrinsic property of NiMnSb, but requires $\sim 5 \%$ excess manganese. ${ }^{18}$ This development motivates a detailed study of the possible configurations of the excess manganese (the vacant position in the $C 1_{b}$ structure but also interchanges with its neighboring atoms), how the moment of the excess manganese couples with the host magnetization, and how it causes the low temperature anomaly. We report on measurements of the magnetization of $\mathrm{NiMnSb}$ with excess manganese at low temperature as well as calculations of electronic structure of $\mathrm{NiMnSb}$ with excess manganese using state-of-the-art first-principle calculations.

\section{DETAILS OF CALCULATIONS, SAMPLE SYNTHESIS, AND MAGNETIZATION MEASUREMENT}

The calculations were carried out using the density functional method in both the local density approximation $(\mathrm{LDA})^{19}$ and the generalized gradient approximation (GGA). ${ }^{20}$ Projector-augmented plane waves are employed ${ }^{21,22}$ as implemented in the Vienna ab initio simulation package (VASP) ${ }^{23-26}$ The kinetic energy cutoff is set to $368 \mathrm{eV}$ and the Brillouin zone of the supercell is sampled with a $k$ mesh of $4 \times 4 \times 8$ (for density of states plots we use a $k$ mesh of $6 \times 6 \times 12$ ). The lattice constant is kept fixed at the experimental value of $5.9268 \AA$ (the GGA functional underestimates the experimental lattice parameter of the primitive cell only by $0.2 \%$ ). The atomic positions are relaxed to a residual force of $0.005 \mathrm{eV} / \AA$ and the criterion for energy convergence is $0.01 \mathrm{meV}$. For calculations with WIEN2K, ${ }^{27}$ we used the GGA $(\mathrm{PBE})^{28}$ exchange-correlation functional. An energy cutoff of $R_{\mathrm{mt}} K_{\max }$ of 8 and a $k$ mesh of $6 \times 6 \times 12$ for Brillouin zone integration were used. $5 \times 10^{-5} \mathrm{Ry}$ for energy and $5 \times 10^{-5} \mathrm{e}$ for charge were used as convergence criteria, while $10^{-4}$ Ry for energy, $10^{-4} \mathrm{e}$ for charge, and $10^{-4} \mathrm{Ry} / a_{0}$ ( $a_{0}$ is the Bohr radius) for force were used in optimization on the positional parameters. The supercell with one excess manganese $\left(\mathrm{Ni}_{16} \mathrm{Mn}_{17} \mathrm{Sb}_{16}\right)$ used in the calculation contains $2 \times 2 \times 1$ conventional cells. Both antiferromagnetic and ferromagnetic ordering between excess manganese and host magnetization were investigated. Sample $\mathrm{NiMn}_{1.05} \mathrm{Sb}$ was synthesized according to the procedure described in Ref. 18. Magnetization was measured in 
TABLE I. Calculated energies of $\mathrm{NiMn}_{1.06} \mathrm{Sb}$, with the excess Mn occupying the vacant position, and interchanging with its nearest neighboring $\mathrm{Sb}, \mathrm{Ni}$, and host $\mathrm{Mn}$ (unit: $\mathrm{eV}$, the energy of the ground state is chosen as 0 ).

\begin{tabular}{|c|c|c|c|c|}
\hline & $\begin{array}{l}\text { Vacant } \\
\text { position }\end{array}$ & $\begin{array}{l}\text { Interchange } \\
\text { with } \mathrm{Sb}\end{array}$ & $\begin{array}{c}\text { Interchange } \\
\text { with } \mathrm{Ni}\end{array}$ & $\begin{array}{l}\text { Interchange } \\
\text { with host Mn }\end{array}$ \\
\hline $\begin{array}{l}\text { Antiferromagnetic } \\
\text { coupling }\end{array}$ & 0 & 3.60 & 0.24 & $0.55^{\mathrm{a}}$ \\
\hline $\begin{array}{l}\text { Ferromagnetic } \\
\text { coupling }\end{array}$ & 0.12 & 3.10 & not obtained & 0.12 \\
\hline
\end{tabular}

${ }^{a}$ The moment on the excess $\mathrm{Mn}$ is antiparallel to that of the host.

a Quantum Design MPMS-XL 7 magnetometer. A little bar shaped sample was cut from an ingot. The sample was placed in a gelatin capsule and fixed in place with cotton. Magnetization versus temperature was measured on heating in different fields, after cooling with $2 \mathrm{~K} / \mathrm{min}$ in a magnetic field of $20 \mathrm{kOe}$. During the measurement a ramp rate of $5 \mathrm{~K} / \mathrm{min}$ was used.

\section{RESULTS AND DISCUSSION}

$\mathrm{NiMnSb}$ crystallizes in the $C 1_{b}$ (half-Heusler) structure, an fcc structure with the $(0,0,0),(1 / 4,1 / 4,1 / 4)$, and $(3 / 4$, $3 / 4,3 / 4$ ) positions occupied by $\mathrm{Ni}, \mathrm{Mn}$, and $\mathrm{Sb}$, respectively. The vacant position $(1 / 2,1 / 2,1 / 2)$ is an obvious candidate for the excess manganese. We also consider interchanges of the excess manganese with its neighboring $\mathrm{Ni}, \mathrm{Sb}$, and host Mn atoms with distance shorter than $3 \AA$. Both ferromagnetic and antiferromagnetic coupling of the excess Mn with respect to the host magnetization were calculated. The results are shown in Table I: The case where excess Mn occupies the vacant position and couples antiferromagnetically to the host Mn has the lowest energy, while in the case of ferromagnetic coupling the energy is higher by $0.12 \mathrm{eV}$. Other cases where the excess $\mathrm{Mn}$ interchanges with $\mathrm{Ni}, \mathrm{Sb}$, and the host $\mathrm{Mn}$ are all higher in energy. The case where the excess $\mathrm{Mn}$ interchanges with $\mathrm{Ni}$ and couples ferromagnetically to the host magnetization is not stable and this solution is not obtained in our calculation. From Table I, we conclude that the excess Mn occupies the vacant position in the supercell, and couples antiferromagnetically to the host $\mathrm{Mn}$ atoms. This conclusion also agrees well with calculations using different codes and exchange-correlation functionals (results are shown in Table II). The energy difference obtained using the LDA functional is smaller compared with those using the GGA

TABLE II. Energy difference (given by $\mathrm{E}_{\text {anti }}-\mathrm{E}_{\text {ferro }}$ ), and the position of Fermi level with respect to the top of the valence bands for the ground state in two codes with different exchange-correlation functionals (unit: $\mathrm{meV}$ ).

\begin{tabular}{lcccc}
\hline \hline & $\begin{array}{c}\text { GGA (PW91) } \\
\text { in VASP }\end{array}$ & $\begin{array}{c}\text { GGA (PBE) } \\
\text { in VASP }\end{array}$ & $\begin{array}{c}\text { LDA in } \\
\text { VASP }\end{array}$ & $\begin{array}{c}\text { GGA (PBE) } \\
\text { in WIEN2K }\end{array}$ \\
\hline $\begin{array}{l}\text { Energy } \\
\text { difference }\end{array}$ & -118 & -118 & -35 & -127 \\
$\begin{array}{l}\text { Position of } \\
\text { Fermi level }\end{array}$ & 36 & 29 & 37 & 40 \\
\hline \hline
\end{tabular}

functional; using GGA functional (both in WIEN2K and VASP), the energy differences are quite similar. In order to avoid being trapped in a local minimum, optimization on the positional parameters should start with some (tiny) distortion on the neighboring atoms of the extra manganese in the supercell, otherwise we find the case where the excess manganese ferromagnetically couples to the host magnetization only.

In a half metal, the position of the Fermi level in the band gap is determined by the metallic spin direction, unlike the situation in a semiconductor, where impurities and surface effects play an important role. The position of the Fermi level with respect to the edges of the band gap is an important quantity determining the stability of the half-metallic properties at finite temperature. Consider a half metal with a band gap for the minority spin direction. At $0 \mathrm{~K}$, the Fermi level intersects the metallic bands for one spin channel, and is located somewhere in the band gap for the semiconducting spin direction. This location is of importance: It determines the robustness of the band gap at finite temperature. For example, consider a case where the Fermi level is close to the top of the valence band (minority spin). A thermal excitation to the conduction band (majority spin) at the Fermi level increases the magnetization, leading to an increase of the exchange splitting (which implies a lowering of the majority spin bands with respect to the minority ones). Consequently a second excitation requires less energy and an avalanche effect occurs at temperatures much below the one corresponding to the energy of the initial excitation. It is important to note that a similar situation occurs if the Fermi level were positioned near the bottom of the conduction band (minority spin): The excitation of a majority spin electron to the bottom of the conduction band decreases the magnetization, leading to the decrease of the exchange splitting (which implies a rising of the majority spin bands with respect to the minority ones), and renders a second excitation more likely. The stable situation is where the effects of both type of excitations cancel: the position of the Fermi level in the middle of the band gap. The behavior of half metals with a band gap for the majority spin is identical: Excitations from the top of the valence bands (majority spin) to conduction bands (minority spin) reduce the magnetization (causing a rising of the majority spin bands with respect to the minority ones), while excitations from the minority spin to the bottom of the conduction band (majority spin) increase the exchange splitting (causing a lowering of the majority spin bands with respect to the minority ones). Detailed knowledge on the DOS (density of states) for $\mathrm{NiMn}_{1.06} \mathrm{Sb}$ close to the Fermi level is an essential ingredient for the understanding of the low-temperature anomaly.

Figure 1 shows the calculated DOS in the vicinity of the Fermi energy for $\mathrm{NiMnSb}$ and $\mathrm{NiMn}_{1.06} \mathrm{Sb}$. The left curve shows the majority spin for pristine NiMnSb. It has a fairly constant DOS deprived of any van Hove singularities, typical for a wide-band, free-electron-like material. The second curve is the corresponding minority spin, and it is typical for a III-V semiconductor. Some van Hove singularities occur in both valence and conduction bands, within the energy range of $1 \mathrm{eV}$ with respect to the Fermi energy. The next two curves show the DOS for the minority spin of $\mathrm{NiMn}_{1.06} \mathrm{Sb}$. The black, continuous curve corresponds to the ground state where the excessive manganese couples antiferromagnetically 


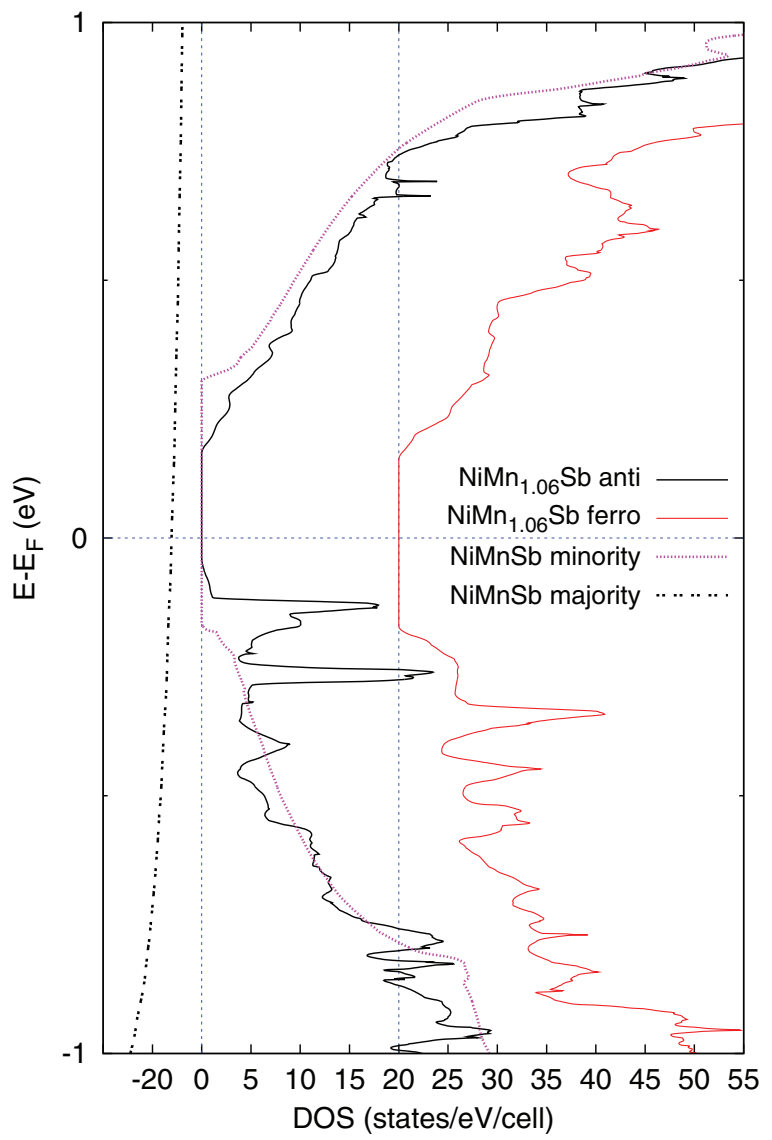

FIG. 1. (Color online) Density of states of $\mathrm{NiMnSb}$ and $\mathrm{NiMn}_{1.06} \mathrm{Sb}$ as function of the energy in the vicinity of the Fermi energy. From left to right: the dotted black curve stands for majority spin for pure $\mathrm{NiMnSb}\left(\mathrm{Ni}_{16} \mathrm{Mn}_{16} \mathrm{Sb}_{16}\right)$, pink curve stands for minority spin for pure $\mathrm{NiMnSb}\left(\mathrm{Ni}_{16} \mathrm{Mn}_{16} \mathrm{Sb}_{16}\right)$, black curve stands for minority spin for $\mathrm{NiMn}_{1.06} \mathrm{Sb}\left(\mathrm{Ni}_{16} \mathrm{Mn}_{17} \mathrm{Sb}_{16}\right)$ with excess manganese orders antiferromagnetically with respect to the host manganese atoms, red curve (which is shifted by 20 states/eV/cell) stands for minority spin for $\mathrm{NiMn} n_{1.06} \mathrm{Sb}\left(\mathrm{Ni}_{16} \mathrm{Mn}_{17} \mathrm{Sb}_{16}\right)$ with ferromagnetic ordering. The accuracy and resolution of all curves are identical. The left curve has a different scale in order to improve the visibility.

to the host manganese atoms, and the red, continuous curve corresponds to the case where they couple ferromagnetically. The configuration with ferromagnetic coupling is $118 \mathrm{meV}$ (shown in Table I) higher in energy compared with the antiferromagnetic case. The position of the Fermi level with respect to the edges of the band gap in the case where the excess manganese orders ferromagnetically to the host is noteworthy: It is exactly in the middle of the gap. So, if this configuration were the ground state, no low-temperature anomaly would be present, independent of the presence of excess manganese. A substantial reduction of the band gap ( $0.20 \mathrm{eV}$ compared to $0.46 \mathrm{eV}$ for pristine NiMnSb) occurs for the antiferromagnetic case, and the position of the Fermi level is just $36 \mathrm{meV}$ (corresponding to a temperature of $418 \mathrm{~K}$ ) with respect of the top of the valence band, which renders the avalanche feedback mechanism possible. Moreover, we find that the position of the Fermi level above the top edge of the valence bands (minority spin) is little affected by different codes and exchange-correlation functionals. ${ }^{29}$ Electrons are thermally excited from the top of the valence bands (minority spin) to the metallic conduction bands (majority spin), and ultimately, the avalanche feedback ends with a half-metalmetal transition. In a normal metal, the temperature scaling of the resistivity is different from that in a half metal. This agrees with the finding in Ref. 18 that the resistivity anomaly starts around $50 \mathrm{~K}$ in the sample with composition $\mathrm{NiMn}_{1.05} \mathrm{Sb}$. This half-metal-metal transition gives the explanation of the anomalous behavior of the resistivity and the loss of high spin polarization in the low-temperature anomaly. In some semiconductors, a somewhat comparable situation ${ }^{30}$ occurs, where thermal excitations of electrons to the conduction band leave holes behind. The electron-hole attraction effectively reduces the band gap, making subsequent excitations more likely and ultimately leads to a transition to a metallic state, the Falicov-Kimball transition. ${ }^{31}$ Here, an enhancement factor of 5 or 6 ( $418 \mathrm{~K}$ versus the temperature around which the lowtemperature anomaly happens) is needed in order to explain the low-temperature anomaly, which is a reasonable number, compared to the enhancement factors in some semiconductormetal transitions. ${ }^{31}$

Next we consider the magnetization in $\mathrm{NiMn}_{1.05} \mathrm{Sb}$. Figure 2 shows the measured magnetization as a function of

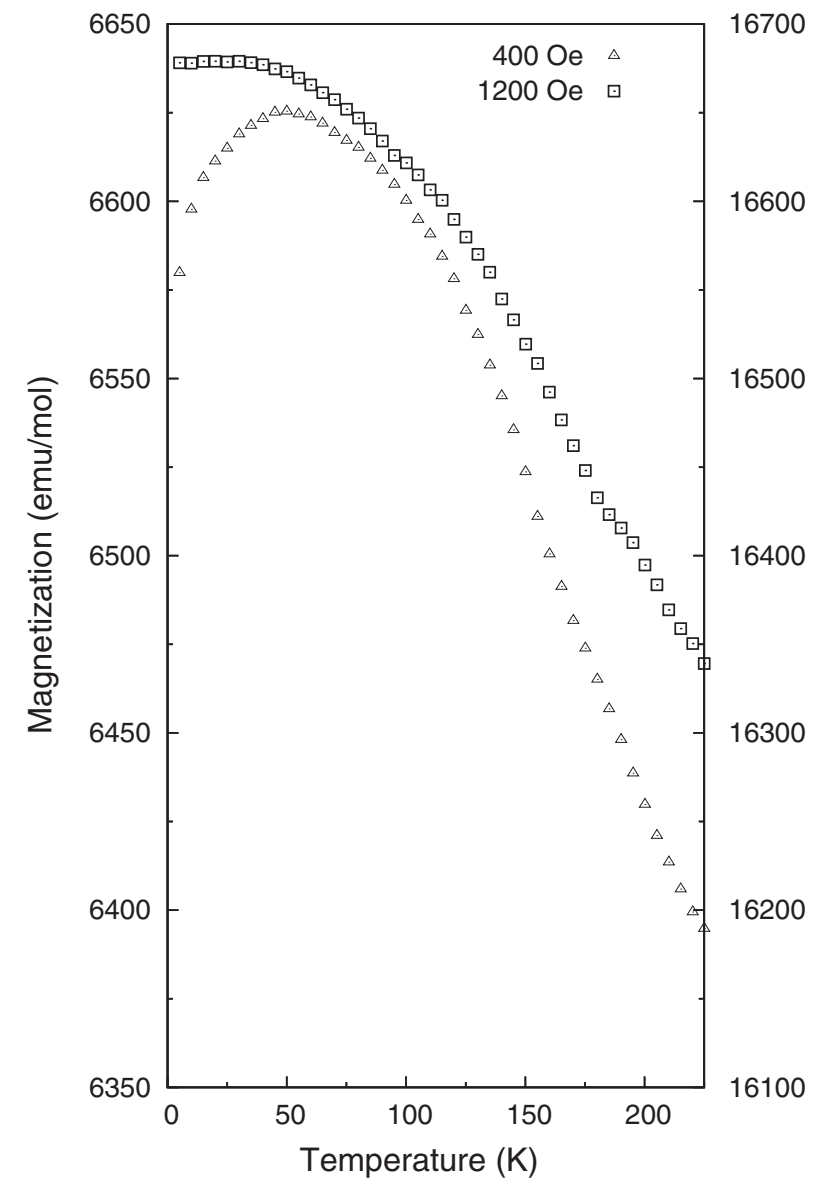

FIG. 2. Magnetization of $\mathrm{NiMn}_{1.05} \mathrm{Sb}$ as function of the temperature from 5 to $225 \mathrm{~K}$ : The curve (scaled by the left vertical axis) plotted by triangles represents magnetization measured in a field of $400 \mathrm{Oe}$, and the curve (scaled by the right vertical axis) plotted by rectangles is for magnetization measured in a field of 1200 Oe. 
the temperature ${ }^{32}$ for two different applied magnetic fields. In a magnetic field of $1200 \mathrm{Oe}$, it behaves like a normal ferromagnet. The behavior in a magnetic field of $400 \mathrm{Oe}$ is quite different: It shows a gradual increase of the magnetization with temperature, leading to a maximum at $50 \mathrm{~K}$. At higher temperatures, the magnetization decreases as is to be expected for a normal ferromagnet. The magnetic anisotropy in $\mathrm{NiMnSb}$ is very small (equivalent to $0.04 \mathrm{~K}$ per magnetic atom), ${ }^{9}$ so this local maximum cannot be related to it. This local maximum can be understood in the following way: When the magnetic filed is $400 \mathrm{Oe}$, at $0 \mathrm{~K}$, moment on the excess manganese couples antiferromagnetically to the host magnetization; as temperature increases, moments on the excess manganese become disordered, so the magnetization is increasing, and this results in a peak centered around $50 \mathrm{~K}$ in the magnetization-temperature curve. This is in perfect agreement with the conclusion in our calculations that the excess manganese couples antiferromagnetically to the host magnetization. A field of $400 \mathrm{Oe}$ is not strong enough to align the antiferromagnetic moments on the excess manganese parallel to the host magnetization, but a field of 1200 Oe can reorient them, even if this field is smaller than the saturation field for $\mathrm{NiMn}_{1.05} \mathrm{Sb}$ which is about $10 \mathrm{kOe}$. We attribute the increase of the magnetization (in a field of $400 \mathrm{Oe}$ ) with temperature to the disordering of the magnetic moments of the interstitial manganese. The antiferromagnetic coupling between the excess manganese and the host magnetization is confirmed by the magnetization analysis. As temperature increases, moments on the excess manganese become disordered. Consequently, this has a distinct influence on the temperature dependence of the magnetization, which gives the explanation of the anomalous behavior of magnetization in the low-temperature anomaly.

\section{CONCLUSIONS}

The experimentally observed anomalous behavior of resistivity and magnetization, and loss of the high spin polarization of the conduction electrons in the low-temperature anomaly in $\mathrm{NiMnSb}$, is not an intrinsic property, but due to the excess manganese. Measurements on the magnetization are in good agreement with the conclusion in our calculations that the excess manganese couples antiferromagnetically to the host magnetization. The half-metal-metal transition is the origin of both the anomaly in resistivity and the loss of high spin polarization; the disordering of moments on the excess manganese gives rise to the anomaly in the magnetization at low temperature. For future work, neutron diffraction experiments on the identification and magnetic properties of the excess $\mathrm{Mn}$, some possible defects in $\mathrm{NiMn}_{1.05} \mathrm{Sb}$, and the magnetization measurement at higher temperature (above $225 \mathrm{~K}$ ) are desirable, and systematic experimental exploration on pure $\mathrm{NiMnSb}$ is expected in order to realize its potential use of half metallicity at higher temperature in spintronics.

\section{ACKNOWLEDGMENTS}

This work is part of the research program of the Foundation for Fundamental Research on Matter (FOM) which is financially supported by the Netherlands Organization for Scientific Research (NWO).
*R.deGroot@science.ru.nl

${ }^{1}$ R. A. de Groot, F. M. Mueller, P. G. van Engen, and K. H. J. Buschow, Phys. Rev. Lett. 50, 2024 (1983).

${ }^{2}$ S. A. Wolf, D. D. Awschalom, R. A. Buhrman, J. M. Daughton, S. von Molnar, M. L. Roukes, A. Y. Chtchelkanova, and D. M. Treger, Science 294, 1488 (2001).

${ }^{3}$ R. A. de Groot, F. M. Mueller, and A. G. M. Janner, Patents NL 19830000602, EP 198402000215 (1983) (discontinued).

${ }^{4}$ K. E. H. M. Hanssen and P. E. Mijnarends, Phys. Rev. B 34, 5009 (1986).

${ }^{5}$ K. E. H. M. Hanssen, P. E. Mijnarends, L. P. L. M. Rabou, and K. H. J. Buschow, Phys. Rev. B 42, 1533 (1990).

${ }^{6}$ G. L. Bona, F. Meier, M. Taborelli, E. Bucher, and P. H. Schmidt, Sol. St. Comm. 56, 391 (1985).

${ }^{7}$ G. A. de Wijs and R. A. de Groot, Phys. Rev. B 64, 020402 (2001).

${ }^{8}$ J. J. Attema, G. A. de Wijs, and R. A. de Groot, J. Phys. D: Appl. Phys. 39, 793 (2006).

${ }^{9}$ C. Hordequin, J. Pierre, and R. Currat, J. Magn. Magn. Mater. 162, 75 (1996).

${ }^{10}$ C. N. Borca, T. Komesu, H. K. Jeong, P. A. Dowben, D. Ristoiu, C. Hordequin, J. P. Nozieres, J. Pierre, S. Stadler, and Y. U. Idzerda, Phys. Rev. B 64, 052409 (2001).

${ }^{11}$ C. Hordequin, D. Ristoiu, L. Ranno, and J. Pierre, Eur. Phys. J. B 16, 287 (2000).
${ }^{12}$ M. Ležaić, P. Mavropoulos, J. Enkovaara, G. Bihlmayer, and S. Blügel, Phys. Rev. Lett. 97, 026404 (2006).

${ }^{13}$ J. J. Attema, G. A. de Wijs, and R. A. de Groot, J. Phys.: Condens. Matter 19, 315212 (2007).

${ }^{14}$ J. J. Attema, C. M. Fang, L. Chioncel, G. A. de Wijs, A. I. Lichtenstein, and R. A. de Groot, J. Phys.: Condens. Matter 16, S5517 (2004).

${ }^{15}$ B. Alling, S. Shallcross, and I. A. Abrikosov, Phys. Rev. B 73, 064418 (2006).

${ }^{16}$ L. Chioncel, E. Arrigoni, M. I. Katsnelson, and A. I. Lichtenstein, Phys. Rev. Lett. 96, 137203 (2006).

${ }^{17}$ T. Graf, C. Felser, and S. S. P. Parkin, Prog. Solid State Chem. 39, 1 (2011).

${ }^{18}$ F. Wang, T. Fukuhara, M. Maezawa, K. Nishimura, F. Shimizu, and S. Masubuchi, Jpn. J. Appl. Phys. 49, 025502 (2010)

${ }^{19}$ W. Kohn and L. J. Sham, Phys. Rev. 140, A1133 (1965).

${ }^{20}$ J. P. Perdew, J. A. Chevary, S. H. Vosko, K. A. Jackson, M. R. Pederson, D. J. Singh, and C. Fiolhais, Phys. Rev. B 46, 6671 (1992).

${ }^{21}$ P. E. Blöchl, Phys. Rev. B 50, 17953 (1994).

${ }^{22}$ G. Kresse and D. Joubert, Phys Rev. B 59, 1758 (1999).

${ }^{23}$ G. Kresse and J. Hafner, Phys. Rev. B 47, 558 (1993).

${ }^{24}$ G. Kresse and J. Hafner, Phys. Rev. B 49, 14251 (1994).

${ }^{25}$ G. Kresse and J. Furthmüller, Comput. Mater. Sci. 6, 15 (1996).

${ }^{26}$ G. Kresse and J. Furthmüller, Phys. Rev. B 54, 11169 (1996). 
${ }^{27}$ P. B. Blaha, K. Schwartz, G. K. H. Madsen, D. Kvasnicka, and J. Luitz, Wien2K, An Augmented Plane Wave Plus Local Orbitals Program For Calculating Crystal Properties (University of Technology, Vienna, 2001).

${ }^{28}$ J. P. Perdew, K. Burke, and M. Ernzerhof, Phys. Rev. Lett. 77, 3865 (1996).

${ }^{29}$ On the position of the Fermi level in the ground state, Table I shows four slightly different values: The smallest value is $29 \mathrm{meV}$ obtained from calculation done in VASP using GGA (PBE) functional, and the largest one is $40 \mathrm{meV}$ obtained from calculation done in WIEN2K using GGA (PBE) functional.
${ }^{30}$ In the half-metal-metal transition, the transfer of an electron from a spin down to a spin up band violates conservation of angular momentum; consequently the real process must be more complex. A priori there is no reason to assume however that these two processes involved differ significantly in efficiency.

${ }^{31}$ L. M. Falicov and J. C. Kimball, Phys. Rev. Lett. 22, 997 (1969).

${ }^{32}$ The sample shows a different color at the surface part compared with the bulk. We report the magnetization measurement on the bulk here; the temperature dependence of the magnetization for the surface part of the sample shows a maximum at higher temperature (around $90 \mathrm{~K}$ ). 